\title{
Radiation-Induced Esophageal Leiomyosarcoma 13 Years after Mediastinal Irradiation Following Surgical Removal of a Thymoma
}

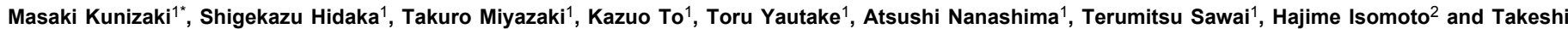
Nagayasu

${ }^{1}$ Department of Surgical Oncology, Nagasaki University Hospital, Sakamoto, Nagasaki 852-8501, Japan

${ }^{2}$ Department of Gastroenterology and Hepatology, Nagasaki University Hospital, Sakamoto, Nagasaki 852-8501, Japan

"Corresponding author: Masaki Kunizaki, Division of Surgical Oncology, Department of Translational Medical Sciences, Nagasaki University Graduate School of Biomedical Sciences, 1-7-1 Sakamoto, Nagasaki 852-8501, Japan, Tel: +95-819-7304; Fax: +95-819-7306; E-mail: makuni49@nagasaki-u.ac.jp

Received date: Mar 26, 2015, Accepted date: Apr 23, 2015, Publication date: Apr 27, 2015

Copyright: ( 2015 Kunizaki M, et al. This is an open-access article distributed under the terms of the Creative Commons Attribution License, which permits unrestricted use, distribution, and reproduction in any medium, provided the original author and source are credited.

\begin{abstract}
Radiation-induced leiomyosarcoma is a very rare complication following irradiation of primary malignancies. In the chest wall, it is usually secondary to radiotherapy for breast cancer or lymphoma. Esophageal malignancy is a radiation dose-related complication of radiotherapy for breast cancer, but its absolute risk is low. A 61-year-old woman presented with an esophageal leiomyosarcoma 13 years after surgery plus mediastinal irradiation (55 Gy) for a stage III thymoma. Esophageal sarcoma is an uncommon tumor, reported sporadically in the literature. Radiotherapy is frequently used in the treatment of carcinoma of the esophagus, and the increased risk of development of sarcoma arising in irradiated fields is well known. The increasing use of radiotherapy for thymoma suggests that radiation-associated malignancies of the esophagus may be seen more frequently in the future. To the best of our knowledge, this is the first report of radiation-induced esophageal leiomyosarcoma after mediastinal irradiation of a thymoma.
\end{abstract}

Keywords: Radiation-induced leiomyosarcoma; Esophageal tumor; Thymoma

\section{Introduction}

Radiation-induced sarcoma (RIS) is a rare late complication after irradiation, with a reported incidence of $0.03 \%-0.8 \%$ [1]. In the chest wall, RIS most commonly follows radiotherapy for breast cancer or malignant lymphoma, appears rare following thymoma. Esophageal leiomyosarcoma is a rare malignant tumor, accounting for less than $1 \%$ of all malignant esophageal tumors [2-4].

Esophageal leiomyosarcoma typically occurs in the middle to distal third of the esophagus because the muscularis propria in that area consists of smooth muscle [5]. These lesions are generally small and asymptomatic, but occasionally can be large and produce dysphagia. Herein, we describe a case of radiation-induced esophageal leiomyosarcoma 13 years after irradiation of a thymoma. To the best of our knowledge, this represents the first such case to be reported.

\section{Case Report}

A 61-year-old woman was referred to hospital with pain and swallowing difficulty. She had undergone surgery for thymoma, which was completely resected together with the adjacent thymus and pericardium at 48 years of age. Adjuvant mediastinal irradiation to the primary site of the tumor using the opposing portal method at a dose of 45 Gy (1.8 Gy x 25) and a further 10 Gy (2 Gy x 5) was administered. Thus, the upper esophagus might have been exposed to a radiation dose of 55 Gy during the overall treatment for thymoma (Figure 1).
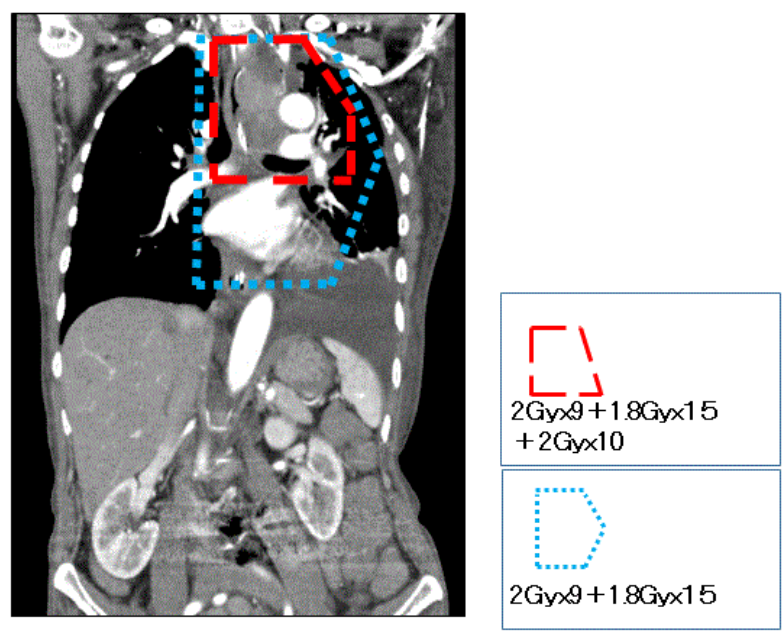

Figure 1: Adjuvant mediastinal irradiation to thethymus using the opposing portal method at a dose of $45 \mathrm{~Gy}$ (1.8 Gy per fraction) and a further $10 \mathrm{~Gy}$ (5 fractions at 2 Gy per fraction) was administered to the upper mediatinal field.

Esophagogastroduodenoscopy showed a stalked intraluminal polypoid mass in the upper thoracic esophagus (Figure 2). The tumor was large enough to fill the esophageal lumen, but allowed passage of a gastrofiberscope (Q260, Olympus, Tokyo, Japan) to the distal part of the esophagus. The specimens taken by endoscopic biopsy were histologically proven to be poorly-differentiated carcinomaand carcinosarcoma was suspected. Computed tomography showed a 
Citation: Kunizaki M, Hidaka S, Miyazaki T, To K, Yautake T, et al. (2015) Radiation-Induced Esophageal Leiomyosarcoma 13 Years after Mediastinal Irradiation Following Surgical Removal of a Thymoma. J Nucl Med Radiat Ther 6: 224. doi:10.4172/2155-9619.1000224

Page 2 of 4

large, well-enhanced soft tissue mass in the upper esophagus with tracheal destruction (Figure 3), but no regional lymph node enlargement or liver metastases. Positron emission tomography (PET)

- computed tomography (CT) showed intense segmental uptake of 18F-fluorodeoxyglucose (FDG) in the upper thoracic esophagus (Figure 4). Accordingly, the patient was diagnosed with esophageal carcinosarcoma (cT3(AD) N0M0 cStage II). The patient underwent transthoracic esophagectomy with three-field lymph node dissection and gastric tube reconstruction. Macroscopically, the resected specimen was a polypoid tumor measuring $6.5 \times 2.7 \mathrm{~cm}$ (Figure 5). Histopathologically, the tumor comprised pleomorphic spindle cells with mitosis and cell necrosis, compatible with leiomyosarcoma. The tumor demonstrated a fascicular growth pattern with spindle-shaped cells demonstrating nuclear atypia and eosinophilic cytoplasm. Mitoses were also frequent, suggesting malignant proliferation (MIB1 $>50 \%$ ). Again, tumor cells stained positively for vimentin and smooth muscle actin (SMA), but negatively for desmin, S-100, CK7, CK20, cKit, and CD34 (Figure 6). Tumor invasion involved the mucosa. Regional lymph nodes were free of metastases. These final findings were consistent with the rare diagnosis of esophageal leiomyosarcoma. No adjuvant radiotherapy or chemotherapy was administered.The patient was discharged home on postoperative day 50 and is currently doing well as of 15 months after surgery.

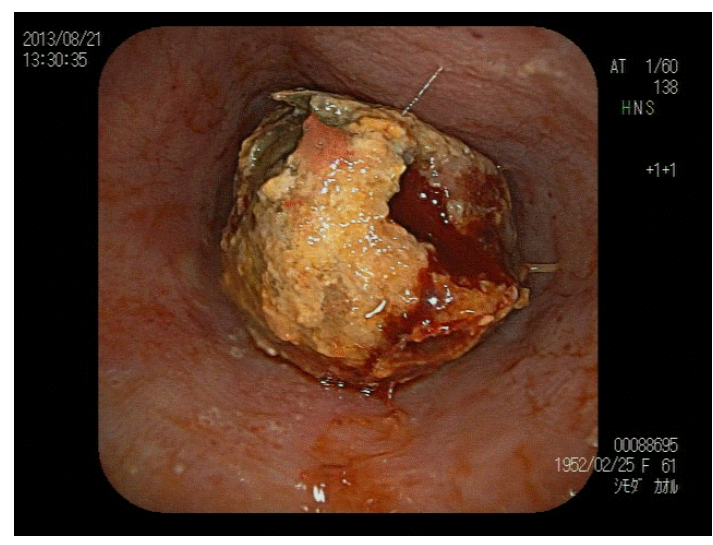

Figure 2: Esophagogastroduodenoscopy shows a large protruding region in the upper esophagus, $25-34 \mathrm{~cm}$ from the incisors.

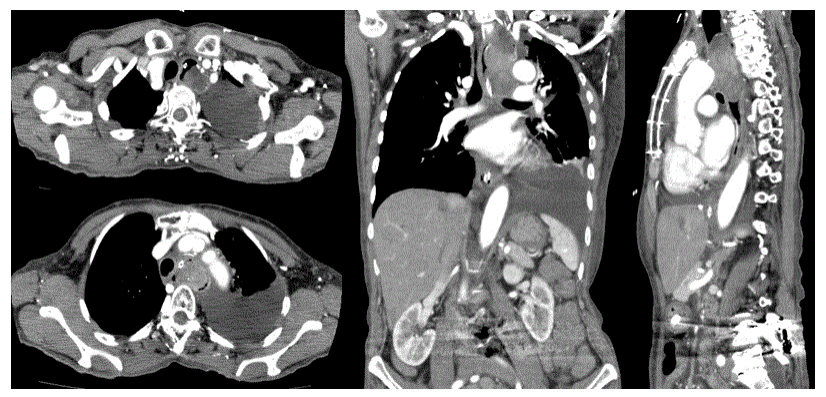

Figure 3: Computed tomography shows a large tumor in the upper thoracic esophagus with tracheal destruction.

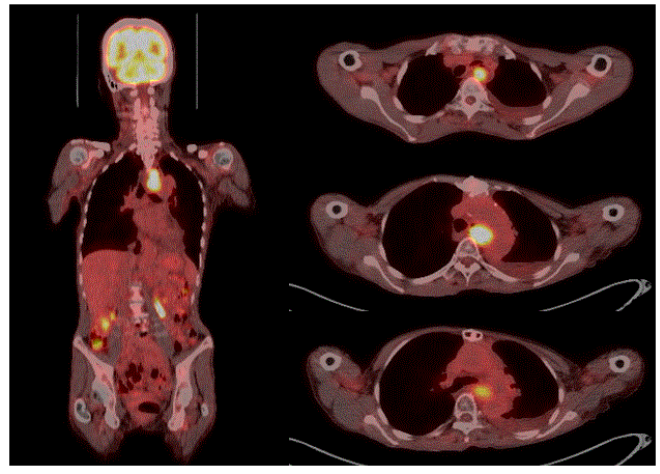

Figure 4: Positron emission tomography-computed tomography shows intense segmental fluorodeoxyglucose uptake at the upper thoracic esophagus.

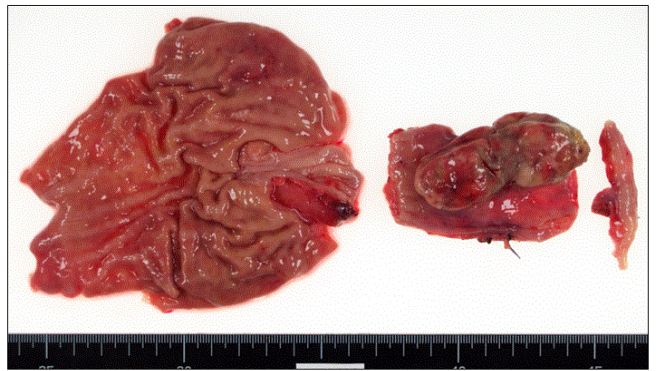

Figure 5: Macroscopic findings of the resected specimen show marked erosion. The resected specimen showed that the mass arising from the submucosa has grown into the lumen and measures about $6.5 \times 2.7 \mathrm{~cm}$.

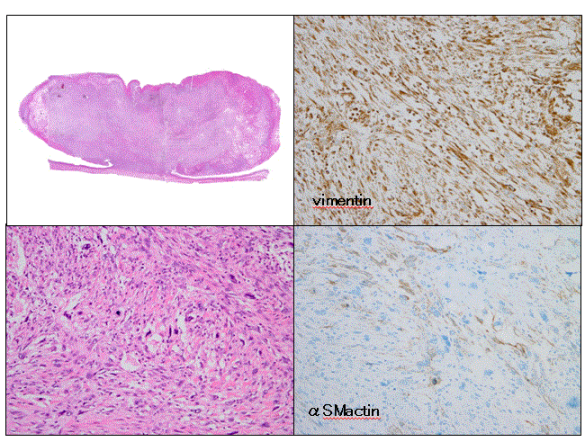

Figure 6: Histological findings of the resected specimen show a spindle cell tumor with cellular polymorphism, mimicking a storiform growth pattern (a). Hematoxylin and eosin staining (b, $\mathrm{x}$ 40). Immunohistochemistry shows positive staining for vimentin and $\alpha$ smooth muscle actin in the cytoplasm of the leiomyosarcoma $(\mathrm{c}, \mathrm{d}, \mathrm{x} 40)$ 


\section{Discussion}

Mesenchymal tumors of the gastrointestinal tract are uncommon. They are usually found in the stomach and small intestine, but can originate throughout the length of the gastrointestinal tract. Those originating from the esophagus are unusual. The most common mesenchymal tumors are the gastrointestinal stromal tumors. These possess smooth muscle features on light microscopy, but in fact originate from interstitial cells derived from mesenchymal stem cells. Finding tumors that actually originate directly from smooth muscle is particularly rare, especially in the esophagus. Such tumors are termed leiomyomas or leiomyosarcomas. Leiomyosarcoma of the esophagus is a rare malignancy, accounting for less than $1 \%$ of all esophageal malignancies [2-4].

Leiomyosarcomas are most commonly located in the middle and lower thoracic esophagus because smooth muscle predominates in that area [5]. Esophageal leiomyosarcomas are typically divided into two types: the polypoid type, representing $60 \%$ of cases; and infiltrative type in $40 \%$ [6,7]. Traditionally, esophagectomy has been considered as the optimal standard management for the disease. However, in a few reports, local excision of some polypoid tumors achieving longterm survival [8]. The prognosis of esophageal leiomyosarcoma is better than that of esophageal squamous cell carcinoma because of its characteristics of slow growth and late metastases $[9,10]$. Patients with polypoid and intramural tumors, tumors in an intrathoracic location, and well-differentiated tumors display better prognoses than patients with infiltrating lesions, tumors in cervical locations, or poorly differentiated tumors $[11,12]$. The present case is unique, because the tumor showed good prognostic factors, such as polypoid type and intrathoracic location, but grew very rapidly and may occurred after radiotherapy. Mediastinal irradiation is known to be a causative factor in esophageal malignancies, but due to the small numbers reported in the literature, no definitive associations between mediastinal radiotherapy and leiomyosarcomas of the esophagus have been made. This represents the first documented case of leiomyosarcoma developing within the esophagus following mediastinal irradiation for thymoma. Many cases of radiation-induced sarcoma have been reported. Although evidence of an association between radiotherapy and leiomyosarcoma remains in sufficients, these cases highlight the possibility of an association, and thus reinforce the importance of maintaining clinical suspicion in post-radiotherapy patients presenting with similar symptoms. The following diagnostic criteria for RIS have therefore been proposed: development in the irradiated field; histological features of RIS differing from the original lesion; and a sufficiently long latent period between radiation and development of neoplasms of over 10 years [13]. The risk of RIS appears to be doserelated, with most cases occurring after receiving doses of at least approximately $55 \mathrm{~Gy}$. The latency period between radiation exposure and development of sarcoma varies from 1.4 to 55 years, with most cases identified between 7 and 14 years [14]. The latency in our case was 13 years, and the radiation dose was $55 \mathrm{~Gy}$. The present patient fulfilled the preceding criteria.

Thymoma is considered radiation-sensitive, and radiotherapy is often used for local control of the tumor. Mediastinal irradiation has been used not only for patients with unresectable tumors, but also as adjuvant therapy for those with Masaoka clinical stage III disease. However, the therapeutic value of adjuvant radiotherapy after complete resection of the tumor has still not been definitively established. Nonetheless, the 10 -year survival reportedly exceeds $80 \%$ if stage III thymomas can be completely resected [15]. Furthermore, immunodeficiency can occur both in patients undergoing radiation therapy, as well as in patients who have had thymectomies [16]. Because many patients achieve long-term survival, even with advanced-stage thymomas, irradiation carries a long-term risks of RIS and immunodeficiency, and such patients therefore need long-term surveillance.

In conclusion, we have described an extremely rare case of radiation-induced esophageal leiomyosarcoma after irradiation of a thymoma. A significant increase has been noted in the number of long-term cancer survivors, in part thanks to recent advances in radiotherapy. Careful long-term surveillance is thus needed for patients treated with radiotherapy. In the current studies have shown that the 3D image-guided radiation therapy technique can effectively detect setup errors of patients undergoing RT, thereby reducing planning target volume margins. These advances will further reduce the radiation dose to critical organs and may reduce the risk of RIS [17]. In addition, effective treatment strategies for esophageal leiomyosarcoma occurring after irradiation need to be developed.

\section{Conflict of interest}

None of the authors have any conflicts of interest to disclose.

\section{References}

1. Mark RJ, Poen J, Tran LM, Fu YS, Selch MT, et al. (1994) Postirradiation sarcomas. A single-institution study and review of the literature. Cancer 73: 2653-2662.

2. Galandiuk S, Hermann RE, Cosgrove DM, Gassman JJ (1986) Cancer of the esophagus. The Cleveland Clinic experience. Ann Surg 203: 101-108.

3. Almeida JM (1982) Leiomyosarcoma of the esophagus. Chest 81: 761-763.

4. Choh JH, Khazei AH, Ihm HJ (1986) Leiomyosarcoma of the esophagus: report of a case and review of the literature. J Surg Oncol 32: 223-226.

5. Zhang BH, Zhang HT, Wang YG (2014) Esophageal leiomyosarcoma: clinical analysis and surgical treatment of 12 cases. Dis Esophagus 27: 547-551.

6. Hatch GF, Wertheimer-Hatch L, Hatch KF, Davis GB, Blanchard DK, et al. (2000) Tumors of the esophagus. World J Surg 24: 401-411.

7. Patel SR, Anandarao N (1990) Leiomyosarcoma of the esophagus. N Y State J Med 90: 371-373.

8. Rocco G, Trastek VF, Deschamps C, Allen MS, Miller DL, et al. (1998) Leiomyosarcoma of the esophagus: results of surgical treatment. Ann Thorac Surg 66: 894-896.

9. Levine MS, Buck JL, Pantongrag-Brown L, Buetow PC, Hallman JR, et al. (1996) Leiomyosarcoma of the esophagus: radiographic findings in 10 patients. AJR Am J Roentgenol 167: 27-32.

10. Camishion RC, Gibbon JH Jr, Templeton JY 3rd (1961) Leiomyosarcoma of the esophagus: review of the literature and report of two cases. Ann Surg 153: 951-956.

11. Rocco G, Trastek VF, Deschamps C, Allen MS, Miller DL, et al. (1998) Leiomyosarcoma of the esophagus: results of surgical treatment. Ann Thorac Surg 66: 894-896.

12. Koga H, Iida M, Suekane H, Aoyagi K, Yao T, et al. (1995) Rapidly growing esophageal leiomyosarcoma: case report and review of the literature. Abdom Imaging 20: 15-19.

13. Cahan WG, Woodard HQ, Higinbotham NL, Stewart FW, Coley BL (1998) Sarcoma arising in irradiated bone: report of eleven cases. 1948. Cancer 82: 8-34.

14. Miller PR, Jackson SL, Pineau BC, Levine EA (2000) Radiation-induced gastrointestinal stromal sarcoma of the esophagus. Ann Thorac Surg 70: 660-662. 
Citation: Kunizaki M, Hidaka S, Miyazaki T, To K, Yautake T, et al. (2015) Radiation-Induced Esophageal Leiomyosarcoma 13 Years after Mediastinal Irradiation Following Surgical Removal of a Thymoma. J Nucl Med Radiat Ther 6: 224. doi:10.4172/2155-9619.1000224

Page 4 of 4

15. Kadota Y, Utsumi T, Inoue M, Sawabata N, Minami M, et al. (2010) Radiation-induced osteosarcoma 17 years after mediastinal irradiation following surgical removal of thymoma. Gen Thorac Cardiovasc Surg 58: 651-653.

16. Wickemeyer JL, Sekhsaria S1 (2014) Prolonged severe immunodeficiency following thymectomy and radiation: a case report. J Med Case Rep 8: 457.
17. Zhang WZ, Chen JZ, Li DR, Chen ZJ, Guo H, et al. (2014) Simultaneous modulated accelerated radiation therapy for esophageal cancer: a feasibility study. World J Gastroenterol 20: 13973-13980. 